\title{
Percutaneous ultrasound guided radiofrequency and microwave ablation in the treatment of hepatic metastases. A monocentric initial experience.
}

\author{
Zeno Sparchez ${ }^{1,2}$, Tudor Mocan ${ }^{1,2}$, Nadim Al Hajjar ${ }^{2,3}$, Adrian Bartos ${ }^{2,3}$, Claudia Hagiu ${ }^{1,2}$, \\ Daniela Matei ${ }^{1,2}$, Rares Craciun ${ }^{2}$, Lavinia Patricia Mocan ${ }^{4}$, Mihaela Sparchez ${ }^{5}$, Daniel \\ Corneliu Leucuta ${ }^{6}$
}

\begin{abstract}
${ }^{1} 3^{\text {rd }}$ Medical Department, "Iuliu Hatieganu" University of Medicine and Pharmacy, ${ }^{2}$ Institute for Gastroenterology and Hepatology, ${ }^{3}{ }^{\text {rd }}$ Surgical Department, "Iuliu Hatieganu" University of Medicine and Pharmacy, ${ }^{4}$ Histology Department, "Iuliu Hatieganu" University of Medicine and Pharmacy, ${ }^{5}{ }^{\text {nd }}$ Paediatric Clinic, ${ }^{6}$ Medical Informatics and Biostatistics Department, "Iuliu Hatieganu" University of Medicine and Pharmacy, Cluj-Napoca, Romania
\end{abstract}

\begin{abstract}
Aim: Percutaneous radiofrequency (RFA) and microwave ablation (MWA) are currently the best treatment options for patients with liver metastases (LM) who cannot undergo a liver resection procedure. Presently, few studies have evaluated the efficacy of tumor ablation in beginner's hands but none at all in hepatic metastasis. Our aim was to report the initial experience with ultrasound as a tool to guide tumor ablation in a low volume center with no experience in tumor ablation. Material and methods: We conducted a retrospective cohort study, on a series of 61 patients who had undergone percutaneous US-guided ablations for 82 LM between 2010 and 2015. Long term outcome predictors were assessed using univariate and multivariate analysis. Results: Complete ablation was achieved in $86.9 \%$ of cases (53/61). All MWA sessions (20/20) attained ablation margins $>5 \mathrm{~mm}$, compared to $79 \%(49 / 62)$ for RFA sessions $(\mathrm{p}=0.031)$. Ablation time was significantly shorter for MWA, with a median duration of 10 minutes (range: 6-12) vs. 14 minutes (range: 10-19.5, p=0.003). There was no statistically significant difference in local tumor progression (LTP)-free survival rates between MWA and RFA ( $\mathrm{p}=0.154)$. On univariate analysis, significant predictors for local recurrence were multiple metastases $(p=0.013)$ and ablation margins $<5 \mathrm{~mm}(\mathrm{p}<.001)$, both retaining significance on multivariate analysis. Significant predictors for distant recurrence on both univariate and multivariate analysis were multiple metastases $(p<0.001)$ and non-colorectal cancer metastases $(p<0.05)$. Conclusion: A larger than 5 mm ablation size is critical for local tumor control. We favor the use of MWA due to its ability to achieve ablation in significantly shorter times with less incomplete ablations.
\end{abstract}

Keywords: colorectal cancer metastases; radiofrequency ablation; microwave ablation

\section{Introduction}

Metastatic liver tumors are linked to an increase in mortality and morbidity. The clinical outcomes of patients with liver metastases have greatly improved in the

Received 20.03.2019 Accepted 10.06.2019

Med Ultrason

2019, Vol. 21, No 3, 217-224

Corresponding author: Dr. Tudor Mocan

Institute for Gastroenterology and Hepatology,

19-21 Croitorilor street, Cluj-Napoca, Romania,

E-mail: mocan_tudor@yahoo.com

Phone: +40799861946 past decades, especially in the field of colorectal cancer liver metastases (CRCLm) [1]. Complete surgical removal is considered the "gold standard" for treating CRCLm [1]. Unfortunately, only $10-20 \%$ have resectable disease at the time of presentation [2]. The vast majority of them cannot benefit from surgical resection either because of deeply located lesions, insufficient hepatic reserves, comorbidities, or the presence of extrahepatic disease [3]. In these circumstances, several ablation methods, including radiofrequency ablation (RFA) and microwave ablation (MWA) have been developed. Both can be performed either directly (intraoperative approach) [4], or 
via percutaneous approach (using computer tomography or ultrasound guidance) [5]. The main advantages of MWA and RFA as opposed to surgical resection reside in their minimally invasive character, repeatability, and a more safety profile [6]. A large number of studies evaluated the outcomes of RFA in both primary and secondary liver tumors $[7,8]$. In patients with unresectable CRCLm, RFA proved to be more effective than chemotherapy alone, with a 5-year survival range between 12 and $48 \%$ [8]. Being simple and safe, RFA has been proposed as an alternative to hepatic resection for small liver metastasis [5]. Only a small number of studies have evaluated the role of MWA in CRCLm with a 4-year overall survival of $35.2 \%$ [9]. There are several theoretical advantages of MWA compared to RFA: shorter ablation times, larger ablation areas, no heat sink effect and no need for grounding pads, but most of these benefits have yet to be consistently proven in human studies [10]. In clinical practice, when comparing RFA and MWA in CRCLm, conflicting results have been reported. One study, using an intraoperative approach found a better local tumor progression (LTP)-free survival for MWA as opposed to RFA [4]. Controversially, a more recent study [5] using CT guidance found no differences in terms of LTP-free survival between those two methods. Additionally, more and more data showed the high efficacy of MWA and RFA in non-CRCLm [11-15]. Only a minority of studies has used conventional ultrasound (provided by medium or low quality US systems) as a guiding tool for tumor ablation in hepatic metastases and the majority of them were conducted in tertiary high volume centers with a vast experience in tumor ablation at the beginning of the studies.

The purpose of this retrospective study was to report the initial experience with US as a tool to guide metastasis ablation in a center with less experience in tumor ablation. Besides investigating the local efficacy, we focused to identify prognostic parameters of LFP-free survival and distant recurrence in patients with hepatic metastases treated with MWA and RFA and also to analyze the complications rate.

\section{Materials and methods}

We conducted a retrospective cohort study, on a consecutive series of patients with hepatic metastases in a single tertiary hospital. Patients were not required to give informed consent to the study because the analysis used anonymous clinical data that were obtained after each patient agreed to treatment by written consent. The study was approved by our Institutional Review Board.

The criteria of patient selection in the present study were the following: tumor detectable by US, single me- tastases $<5 \mathrm{~cm}$ in maximum diameter or multi-nodular (up to three in number) $\leq 3 \mathrm{~cm}$ in maximum diameter each, tumors accessible via percutaneous approach at US, prothrombin time ratio $>50 \%$, and a platelet count $>70.000$ cells $/ \mathrm{mm}^{3}$. Closed proximity to the colon, gallbladder or hepatic hilum ware considered contraindications for ablation.

Between January 2010 and December 2015, 78 consecutive patients with hepatic metastases were referred to our institution for percutaneous ablation therapy (PAT). Five patients were excluded because they were treated either by intraoperative MWA $(\mathrm{n}=2)$ or intraoperative RFA ( $n=3)$. Among the remaining 73 patients, another set of 12 patients treated with RFA was excluded due to incomplete data $(\mathrm{n}=8)$ or a follow-up shorter than 3 months $(n=4)$. All patients were considered to be clear of hepatic metastases at the initial curative-intent surgery with R0 resection, but subsequently become symptomatic on follow-up and were diagnosed with hepatic metastases on radiological images. A multimodal approach, including surgery, systemic chemotherapy and/or radiotherapy depending on tumor type, was used at the time of primary tumor diagnosis. The reasons for not performing surgical resection of the metastases were: previous hepatectomy $(n=7)$, extrahepatic disease $(n=4)$, comorbidities $(n=22)$, patient choice $(n=17)$, disease being deemed technically unresectable $(n=5)$, and the presence of a solitary deep tumor requiring major resection $(n=6)$. Prior to treatment, all patients underwent imaging studies including US, contrast enhanced computed tomography (CECT) and/or magnetic resonance imaging (MRI), physical examination, and laboratory tests.

\section{RFA and MWA procedures}

All procedures were performed percutaneously in the surgical room under local anesthesia and intravenous sedation using US guidance (Aloka Prosound SSD 3500SX system with a $3.5 \mathrm{MHz}$ transducer) by one operator with no experience in tumor ablation at the beginning of the inclusion period but with more than 10 years experience in interventional ultrasound. For RFA treatment a 200 W generator (model 1500X; Rita Medical Systems, AngioDynamics) coupled to an expandable array with nine electrode tines was used. Two grounding pads were attached to the patient's legs. The electrode was inserted into the lesion and deployed to a step between 2 and $5 \mathrm{~cm}$ depending on tumor size and proximity with surrounding structures. When reaching the target temperature $\left(105^{\circ} \mathrm{C}\right)$, the needle tip was continuously perused with sodium chloride by an internal channel inside the needle throughout the ablation to keep the temperature of the tip low, thus preventing charring around. At the end of the procedure, the generator was reactivated and the 
needle track was ablated. The microwave ablation was performed with the Avecure MWG 881 (MedWaves, Inc. San Diego, CA). The system consists of a microwave generator, a flexible low-loss coaxial cable and an antenna. The output power was set at $32 \mathrm{~W}$ and an $14 \mathrm{G}$ antenna was used. The frequency is automatically controlled with real-time temperature and reverse-power feedback during ablation to optimize ablation safety, efficiency, energy transfer, and predictability. During ablation, a hyperechoic area was observed at US around the electrode tip; this area increased progressively to cover all of the lesions. Each ablation cycle lasted between 3 and 12 minutes depending on tumor size and number. In the case of the subcapsular tumor, we avoided direct perpendicular puncture and in case of large tumors, multiple overlapping ablations were performed. Both MWA and RFA were performed using the free hand technique. We tried to use MWA in perivascular locations, in lesions close to other structures (gallbladder, biliary ducts, gastric or colonic wall) where the 1 or more hooks of the RF umbrella could produce a thermal injury or in subcapsular locations. In the rest of the lesions the system (RF or MW) was chosen based on the availability of the system at that particular moment. The time of each procedure was recorded (starting at the moment when the tumor temperature reached its target until the end of the treatment). After the treatment, patients were rescanned 60 minutes later to detect any bleeding in the liver or the peritoneal cavity. Rescans were performed by the same operator.

\section{Definitions}

Complete ablation was defined as an ablation defect completely covering the target tumor with no evidence of residual disease on contrast enhanced ultrasound (CEUS) performed 4 weeks after the procedure. All CEUS examinations were performed by the same operator that performed the ablations. In cases of partial ablation an additional session was considered. Dynamic CT scans with section thickness of $5 \mathrm{~mm}$ were performed 3 months after treatment in all patients. Subsequently all patients underwent CECT every 3 months. MRI was obtained for further evaluation of questionable findings. Local tumor progression was defined as a hypodense lesions or peripheral/nodular enhancement less than $1 \mathrm{~cm}$ from the edge of the ablation zone on CECT. Distant recurrences were defined as the emergence of one or several tumor(s) not adjacent to the ablation zone. The observation period for tumor recurrence was defined as the interval between the first RFA and either the detection of tumor recurrence, death, or the last visit before December 31, 2017, whichever came first. Whatever the type of recurrence, additional ablation was considered according to the same criteria as those used at the time of the initial treatment. In cases of incomplete ablation, the local tumor progression was calculated from the second ablation session. Patients with multifocal liver and/or extrahepatic progression were referred for systemic chemotherapy. The ablations margins were measured using the first pre- and post-ablation CEUS. Using CEUS all lesions and all ablation defects were measured in two axes before and one month after treatment. The size of the tumors was compared with the size of the ablation defect. Margin size was classified as: $<5 \mathrm{~mm}$ (if the ablation defect in all axes was less than $5 \mathrm{~mm}$ larger compared to initial tumor size) and $>5 \mathrm{~mm}$ (if the ablation defect in all axes was more than $5 \mathrm{~mm}$ larger compared to initial tumor size). These assessments were done by the same operator that performed the initial CEUS, treatment and one month rescans.

\section{Complications}

Complications were classified as minor (requiring no therapy) and major (requiring therapy and hospitalization) according to Society of Interventional Radiology guidelines [16,17].

\section{Statistical analysis}

The proportion of baseline factors and complications rates were compared using the chi-squared or Fischer exact test. The non-parametric Mann-Whitney U test was used to compare the total ablation time between RFA and MWA groups. Overall survival was defined as the interval between treatment initiation and death. Survival curves were generated by the Kaplan-Meier method and compared with the log-rank test. We defined a session and a treatment according to the working party report on image guided tumor ablation [16]. The prognostic relevance of baseline characteristics was analyzed by univariate and multivariate Cox proportional hazards regression models. All variables taken into account into the univariate analysis were assessed in multivariate analysis. For all regressions, we checked the proportional hazard assumption, and for multivariate analyses we checked the presence of multicollinearity. The results of the Cox analysis were presented as a hazard ratio with corresponding 95\% confidence interval (CI), with $\mathrm{p}$ values based on the Wald statistic. All significance tests were two-tailed, and tests with a $\mathrm{p}$ value $<0.05$ were considered statistically significant. The analyses were performed in $\mathrm{R}$ version 3.2.3 software.

\section{Results}

The baseline characteristics are reported in table I. In total, 61 patients with 82 lesions (median size: 24.88 $\mathrm{mm}$, range $8-44 \mathrm{~mm}$ ) were treated with PAT: 40 patients 
had CRCLm and 31 had non-CRCLm. In all, 45 patients $(73.7 \%)$ had single tumors, and $16(26.3 \%)$ had multiple tumors. When comparing RFA and MWA (Table II) there were no statistically significant differences regarding age, sex, tumor number, tumor size, primary tumor site, number of ablation sessions, median follow-up, complete ablation rate, local recurrence, or distant recurrence. The total ablation time per tumor was, however, significantly shorter in the MWA group ( $\mathrm{p}=0.003)$ with a median of 10 minutes (range: 6-12) versus 14 minutes (range 10-19.5) for RFA. MWA treatment successfully achieved an ablation margin larger than $5 \mathrm{~mm}$ in all tumors compared to RFA treatment where only 49 out of $62(79 \%)$ nodules had an ablation margin $>5 \mathrm{~mm}(\mathrm{p}=0.031)$.

\section{Local recurrence and overall survival}

Overall LTP-free survival at $6,12,18$, and 24 months was $91 \%, 79 \%, 75 \%$ and $67 \%$, respectively. The LTPfree survival rates were lower for RFA versus MWA at 12,24 and 36 months $(73 \%$ versus $93 \%, 60 \%$ versus $85 \%$, and $53 \%$ versus $68 \%$, respectively) however the difference was not statistically significant $(\mathrm{p}=0.154)$. Furthermore, no difference was found between CRCLm and non-CRCLm with a LTP-free survival at 12 months of $83 \%$ versus $72 \%$ ( $\mathrm{p}=0.964)$.

Overall survival was not influenced by the treatment type. The 12 - and $24-$ months' overall survival was $81 \%$ versus $73 \%$ and $67 \%$ versus $61 \%$, respectively between MWA and RFA. Overall survival was instead affected by the type of primary tumor. The 12, 24, and 36 months' overall survival rates were $81 \%$ versus $63 \%, 73 \%$ versus $45 \%$ and $63 \%$ versus $31 \%$ respectively in CRCLm versus non-CRCLm ( $\mathrm{p}=0.025)$.

\section{Univariate and multivariate analysis}

On univariate analysis, predictors of shorter LTP-free survival were an ablation margin of $5 \mathrm{~mm}$ or smaller and more than one tumor (fig 1, Table III). On multivariate analysis, both ablation margin of $5 \mathrm{~mm}$ or smaller $(p=0.002)$ and tumor number $(p=0.049)$ were independent predictors. The LTP rate for tumors with a margin of $5 \mathrm{~mm}$ or smaller and larger than $5 \mathrm{~mm}$ were $76.9 \%$ $(n=10 / 13)$ and $18.8 \%(n=13 / 69)$. The LTP rates for multiple (more than one tumor) versus single tumors were $62.5 \%(n=10 / 16)$ and $21.31 \%(n=13 / 61)$.

On univariate analysis, predictors of distant recurrence free survival were non-colorectal cancer liver metastases and tumor number. On multivariate analysis, both the presence on non-CRCLm $(p=0.001)$ and tumor number greater than one $(\mathrm{p}<0.001)$ were independent predictors (Table IV).

\section{Complications}

The complications of both RFA and MWA and the treatment of each complication is depicted in table V.
Table I. Overall baseline characteristics

\begin{tabular}{ll}
\hline Age (years) & $\mathbf{6 0 . 7 2 \pm 1 0 . 1 3}$ \\
\hline Gender (female) & $29 / 61(47.54)$ \\
Primary tumor & \\
$\quad$ colorectal cancer & $40 / 61(65.57)$ \\
breast & $8 / 61(13.11)$ \\
pancreas & $4 / 61(6.56)$ \\
stomach & $4 / 61(6.56)$ \\
ampuloma & $1 / 61(1.64)$ \\
gall bladder & $1 / 61(1.64)$ \\
lung & $1 / 61(1.64)$ \\
melanoma & $1 / 61(1.64)$ \\
ovary & $1 / 61(1.64)$ \\
Tumor number & \\
1 & $45 / 61(73.77)$ \\
2 & $11 / 61(18.03)$ \\
3 & $5 / 61(8.2)$ \\
Tumor size (mm) & $25(8-44)$ \\
Tumor necrosis (mm) & $38(16-61)$ \\
Prior liver resection (yes/no) & $7 / 61(11.48)$ \\
Treatment of recurrences & \\
systemic chemotherapy & $6 / 28(21.43)$ \\
MWA & $9 / 28(32.14)$ \\
RFA & $12 / 28(42.86)$ \\
surgery & $1 / 28(3.57)$ \\
\hline
\end{tabular}

The results are expressed as mean $\pm \mathrm{SD}$ or number of patients/total number $(\%), \mathrm{mm}=$ millimeters, $\mathrm{MWA}=$ Microwave ablation, RFA $=$ radio-frequency ablation

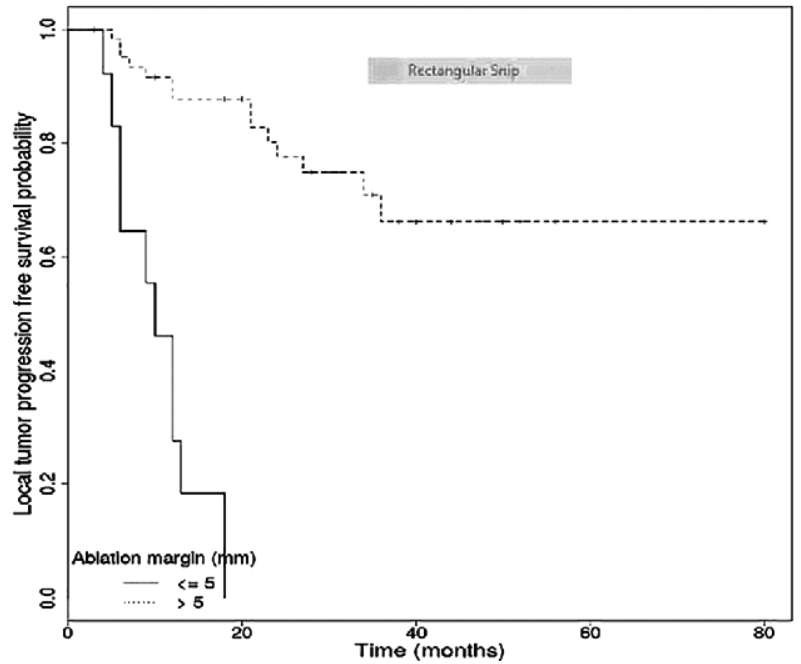

Fig 1. Local tumor progression-free-survival according to the ablation margin.

There was no difference in complication rates between groups $(\mathrm{p}=0.46)$. 


\section{Discussions}

The present study describes our clinical experience with percutaneous ultrasound guided MWA and RFA in the treatment of liver metastases. In total, 127 ablation sessions have been performed in 82 lesions. Complete ablation rate, regardless of the method used was $86.9 \%$.

Table II. Comparison of baseline patient/tumor characteristics

\begin{tabular}{|c|c|c|c|}
\hline Characteristic & MWA group & RFA group & p value \\
\hline Number of tumors & 20 & 62 & \\
\hline Age (years) & $62.12 \pm 10.73$ & $60.18 \pm 9.96$ & 0.508 \\
\hline Gender (Female) & $8(47.06)$ & $21(47.73)$ & 0.963 \\
\hline \multicolumn{4}{|l|}{ Tumor type } \\
\hline ampuloma & $0(0)$ & $1(2.27)$ & \\
\hline breast & $3(17.65)$ & $5(11.36)$ & \\
\hline colorectal cancer & $11(64.71)$ & $29(65.91)$ & \\
\hline gall bladder & $1(5.88)$ & $0(0)$ & 0.466 \\
\hline lung & $1(5.88)$ & $0(0)$ & \\
\hline melanoma & 0 & $1(2.27)$ & \\
\hline ovary & 0 & $1(2.27)$ & \\
\hline pancreas & 0 & $4(9.09)$ & \\
\hline stomach & $1(5.88)$ & $3(6.82)$ & \\
\hline Tumor size (mm) & $25.5(15-33)$ & $25(16.5-30)$ & 0.996 \\
\hline Tumor number $(>1)$ & $2(11.76)$ & $14(31.82)$ & 0.193 \\
\hline \multicolumn{4}{|l|}{ Ablation sessions } \\
\hline 1 & $9(45)$ & $34(54.84)$ & 0.612 \\
\hline 2 & $10(50)$ & $23(37.1)$ & \\
\hline 3 & $1(5)$ & $5(8.06)$ & \\
\hline $\begin{array}{l}\text { Intervention time } \\
\text { (minutes) }\end{array}$ & $10(6-12)$ & $10(10-19.5)$ & 0.003 \\
\hline $\begin{array}{l}\text { Ablation margin } \\
(\leq 5 \mathrm{~mm})\end{array}$ & 0 & $13(20.97)$ & $0.03 *$ \\
\hline Prior liver resection & 0 & $7(15.91)$ & 0.175 \\
\hline
\end{tabular}

In the MWA group the complete ablation rate was significantly higher $(100 \%$ vs. $71.35 \%, p=0.008)$. Compared to other published studies $[5,18]$ we had a slightly lower therapeutic effectiveness. These differences might be explained by differences in terminology and followup protocols. First, we did not consider multiple ablation sessions on the same lesion as a therapeutic success. Secondly, we used a middle range US machine (like those existing in the surgical rooms, intensive care units) for RFA and MWA guidance with medium image resolution (clearly affecting the result of the treatment). We had no possibility to perform CEUS intra-procedurally to detect incomplete ablations. With the expense of increasing the procedure time, the use of CEUS intra-procedurally has clearly increased the rate of complete ablations [19]. One study reported that due to intraprocedural CEUS a second session of treatment was spared in $31.1 \%$ of patients [19]. Third, at the start of the study we had almost no experience with tumor ablation. Nevertheless, when evaluating the learning curve for RFA one study reported a lower complete ablation rate in the first 50 patients as opposed to the next 50 patients $(85.7 \%$ vs. $100 \%, \mathrm{p}=0.006)$ [20]. These findings are similar with ours, despite the fact that we included only patients with hepatic metastases which are more difficult to treat, with a higher rate of recurrence as opposed to HCC [21].

We use CEUS for the first follow-up of our patients rather than CT scans as previously reported $[5,18]$. The use of CEUS is more economical than CT while still being a reliable method to assess the necrosis area or to depict hyper-vascular areas inside the treated lesions in the arterial phase or washout in the late phase [19]. When compared to CT, CEUS was shown to be superior in the first follow-up of patients with hepatic tumors treated with RFA (specificity of 96\% vs. 92\%) [22].

Overall, the local recurrence rate was lower for MWA (12.5\%) compared to RFA (34.88\%) however the difference was not statistically significant. Correa-Gallego et al [4] also found MWA to be superior to RFA in terms of

Table III. Cox survival analysis of predictors for local recurrence in patients with liver metastases after thermal ablation

\begin{tabular}{lllllcc}
\hline & \multicolumn{3}{c}{ Univariate } & \multicolumn{3}{c}{ Multivariate } \\
\cline { 2 - 6 } & HR unadjusted & $95 \%$ CI & $\mathrm{p}$ & HR adjusted & $95 \%$ CI & $\mathrm{p}$ \\
\hline Treatment (RFA/MWA) & 2,46 & $0.71-8.47$ & 0.154 & 1,53 & $0.4-5.7$ & 0.531 \\
Primary tumor (other/colorectal) & 0,98 & $0.35-2.73$ & 0.973 & 1,87 & $0.57-6.08$ & 0.299 \\
Age (years) & 1,01 & $0.96-1.06$ & 0.743 & 1,03 & $0.98-1.08$ & 0.284 \\
Tumor number (single/multiple) & 0,3 & $0.11-0.77$ & $\mathbf{0 . 0 1 3}$ & 0,33 & $0.11-1$ & $\mathbf{0 . 0 4 9}$ \\
Size (mm) & 1,03 & $0.98-1.07$ & 0.218 & 1,01 & $0.96-1.06$ & 0.809 \\
Ablation margin $(>5 \mathrm{~mm} \leq 5 \mathrm{~mm})$ & 0,09 & $0.03-0.28$ & $<\mathbf{0 . 0 0 1}$ & 0,13 & $0.04-0.48$ & $\mathbf{0 . 0 0 2}$ \\
\hline
\end{tabular}

$\mathrm{HR}=$ hazard ratio; $\mathrm{CI}=$ confidence interval, $\mathrm{MWA}=$ microwave ablation, $\mathrm{RFA}=$ radiofrequency ablation, $\mathrm{mm}=$ millimeters; all variables were kept in the multivariable model, $p=$ level of significance 
Table IV. Cox survival analysis of predictors for distant recurrence in patients with liver metastases after thermal ablation

\begin{tabular}{|c|c|c|c|c|c|c|}
\hline & \multicolumn{3}{|c|}{ Univariate } & \multicolumn{3}{|c|}{ Multivariate } \\
\hline & HR unadjusted & $95 \% \mathrm{CI}$ & $\mathrm{p}$ & HR adjusted & $95 \% \mathrm{CI}$ & $\mathrm{p}$ \\
\hline Age (years) & 0.99 & $0.95-1.03$ & 0.576 & 1.01 & $0.97-1.05$ & 0.524 \\
\hline Primary tumor (other/colorectal) & 2.43 & $1.09-5.44$ & 0.031 & 5.06 & $1.9-13.51$ & 0.001 \\
\hline Tumor number (single/multiple) & 0.2 & $0.09-0.45$ & $<0.001$ & 0.1 & $0.03-0.3$ & $<0.001$ \\
\hline Size $(\mathrm{mm})$ & 1.01 & $0.97-1.05$ & 0.765 & 1 & $0.95-1.04$ & 0.827 \\
\hline Treatment (RFA/MWA) & 1.62 & $0.61-4.36$ & 0.335 & 0.97 & $0.33-2.88$ & 0.955 \\
\hline Complete ablation (no/ yes) & 1.96 & $0.57-6.77$ & 0.287 & 1.5 & $0.38-5.95$ & 0.567 \\
\hline
\end{tabular}

$\mathrm{HR}=$ hazard ratio; $\mathrm{CI}=$ confidence interval, $\mathrm{MWA}=$ microwave ablation, $\mathrm{RFA}=$ radiofrequency ablation, $\mathrm{mm}=$ millimeters; all variables were kept in the multivariable model, $p=$ level of significance

Table V. Complications in the RFA and MWA groups

\begin{tabular}{|c|c|c|c|c|c|}
\hline \multicolumn{6}{|l|}{ Major complications } \\
\hline RFA group & & & MWA Group & & \\
\hline Complications (class) & No. & Treatment & Complications (class) & No. & Treatment \\
\hline Abscess & 2 & External drainage & Colonic perforation & 1 & Conservative \\
\hline Perihepatic hematoma & 1 & Blood transfusion & & & \\
\hline Pleural effusion & 1 & Conservative & Pleural effusion & 1 & Conservative \\
\hline Total $n(\%)$ & \multicolumn{3}{|l|}{$4(9.09 \%)$} & \multicolumn{2}{|l|}{$2(10 \%)$} \\
\hline \multicolumn{6}{|l|}{ Minor complications } \\
\hline RFA Group & & & MWA Group & & \\
\hline Complication (class) & No. & & Complication class & No. & \\
\hline Bilioma & 1 & & & & \\
\hline AP fistula & 2 & & & & \\
\hline Total n (\%) & $3(6.8 \%)$ & & & 0 & \\
\hline
\end{tabular}

$\mathrm{MWA}=$ microwave ablation, $\mathrm{RFA}=$ radiofrequency ablation, $\mathrm{AP}=$ arterio-portal, no $=$ number

local recurrence $(6 \%$ versus $20.3 \%)$ but this difference was statistically significant $(\mathrm{p}<0.01)$. The lack of significance observed in our study, may be due to the small samples in the MWA group. In contrast, a more recent study found no difference between MWA and RFA [5]. When comparing our recurrence rate for the MWA group with other studies [9] with high expertise in MWA there is no difference. However, for the RFA group we observed a higher recurrence rate compared to others $[4,5]$ suggesting that MWA is less affected by the operator experience. Moreover, the recurrence rate for the MWA in our study $(12.5 \%)$ is lower than the recurrence rate $(20 \%)$ reported by other highly experienced operators in RFA [4]. This might suggest that the learning curve for MWA is different.

In both univariate and multivariate analysis, margin size was a significant predictor of LTP-free survival, highlighting the importance of achieving sufficient ablation margins. Similar findings were also reported by others [5]. Different from studies which used CECT $[5,23,24]$, we assessed the margin size based on CEUS using a simple algorithm. Indeed, the method we used might be more prone to errors (e.g. no accounting for centering the tumor); however, it is easier to implement and less time-consuming. Despite the fact that, perivascular and subcapsular lesions were preferentially treated with MWA, all lesions treated with MWA achieved a 5 $\mathrm{mm}$ or more margin size as opposed to RFA where only $79 \%$ achieved a secure margin size. Moreover, MWA was shown to produce larger ablation areas in animal studies $[25,26]$. The results presented here validate the findings on animal studies. This is of particular importance since a margin size greater than $10 \mathrm{~mm}$ is rarely associated with LTP [5]. Nevertheless, a margin size $>10 \mathrm{~mm}$ is mandatory in mutant RAS CRCLm as opposed to wild-type RAS CRCLm [27].

We also found the tumor number to be a significant predictor of LTP-free survival on multivariate analysis, a finding similar to others [28]. Two more recent studies $[4,5]$ have found that the tumor number was not associated with LTP-free survival. However, unlike the aforementioned studies we also included non-CRCLm in our study which is more aggressive than CRCLm. Distant recurrence rate in our study was in accordance 
with previous data [8]. Tumor number and the presence of non-CRCLm were significant predictors of distant recurrence. This result might suggest that local recurrence rate is more closely linked to therapeutic success and the choice of treatment, while distant recurrence is largely determined by overall tumor burden, tumor biology and tumor aggressiveness. Although further data is needed, these findings might support the use of thermal ablation in non-CRCLm, especially if the primary tumor is controlled and there is only a single hepatic metastasis.

No difference was observed in the complication rates between the two ablation techniques which are in concordance with other studies $[4,5]$. More importantly, the complication rate in our study was not different from other studies in which the thermal ablation was performed by skilled investigators. One study reported a rate of early complications of $7.1 \%$ [29], similar to the reported rate for RFA in our study (6.8\%). However, the study of Poon et al [20] showed a higher morbidity for patients treated by operators with less experience as opposed to those with a higher experience ( $16 \%$ vs. $4 \%, \mathrm{p}=0.048)$ highlighting the importance of operator experience in patients' outcome.

This study has several limitations. Firstly, the retrospective nature and the lack of data about tumor markers might have influenced the results. Secondly, only a limited number of patients had extrahepatic disease $(n=4)$ or have been previously treated by surgical resection $(n=7)$, hence, we could not include these variables in the univariate or multivariate analysis. Third, the sample size was small, heterogeneous, and the groups were numerically very different.

Despite all the limitations, the study is also coming up with some new insights. This is among the first studies reporting the value of ultrasound as a tool to guide both percutaneous MWA and RFA in various types of hepatic metastases. Furthermore, few studies have evaluated the importance of secure margins. We proposed here a novel and simple method to estimate the ablation margins comparing CEUS images performed before with those after tumor ablation. Another method (commonly used in USA) to estimate the ablation margins could be the use of fusion imaging of CEUS with CT or MRI [24]. Only two studies have evaluated the impact of operator experience in RFA treatment. One showing a higher rate of complete ablation in experienced hands [20] and the other evidenced a worse evolution in less experienced operators [30]. This is of particular importance since our results could be easily extrapolated to other centers that are planning to implement tumor ablation in their departments. The results reported in clinical trials by investigators with high experience in tumor ablation do not always reflect real world data. Nevertheless, when using US as a guiding tool (although more patient friendly and less time consuming compared to CT or MRI) tissue necrosis prevents visualization of needle tip and cannot account for any migrations of the ablation probe. Knowing what to expect from a technique is important before starting to implement it.

In conclusion, we would like to offer some suggestions for other interventional ultrasound departments who are planning to start performing tumor ablation. First, the use of very good US systems with CEUS facilities is mandatory to ensure good results and lower complications. Secondly, the main focus should be to obtain a margin size of at least $5 \mathrm{~mm}$. Thirdly, treating only solitary and small (up to $3 \mathrm{~cm}$ ) hepatic metastases at the beginning might be more appropriate. Nevertheless, choosing between MWA and RFA is also important since both are equally available on the market. From our experience MWA is less time consuming, it might be less affected by operator experience and should be the first choice in centers trying to implement tumor ablation.

\section{Conflict of interest: none}

\section{References}

1. Van Cutsem E, Cervantes A, Adam R, et al. ESMO consensus guidelines for the management of patients with metastatic colorectal cancer. Ann Oncol 2016;27:1386-1322.

2. Ruers T, Bleichrodt RP. Treatment of liver metastases, an update on the possibilities and results. Eur J Cancer 2002;38:1023-1033.

3. Feliberti EC, Wagman LD. Radiofrequency ablation of liver metastases from colorectal carcinoma. Cancer Control 2006; 13:48-51.

4. Correa-Gallego C, Fong Y, Gonen M, et al. A Retrospective Comparison of Microwave Ablation vs. Radiofrequency Ablation for Colorectal Cancer Hepatic Metastases. Ann Surg Oncol 2014;21:4278-4283.

5. Shady W, Petre EN, Do KG, et al. Percutaneous Microwave versus Radiofrequency Ablation of Colorectal Liver Metastases: Ablation with Clear Margins (A0) Provides the Best Local Tumor Control. J Vasc Interv Radiol 2018;29:268275 .

6. Bertot LC, Sato M, Tateishi R, Yoshida H, Koike K. Mortality and complication rates of percutaneous ablative techniques for the treatment of liver tumors: A systematic review. Eur Radiol 2011;2:2584-2596.

7. Verslype C, Rosmorduc O, Rougier P; ESMO Guidelines Working Group. Hepatocellular carcinoma: ESMO-ESDO clinical practice guidelines for diagnosis, treatment and follow-up. Ann Oncol 2012;23 Suppl 7:vii41-vii48.

8. Pathak S, Jones R, Tang JM, et al. Ablative therapies for colorectal liver metastases: A systematic review. Color Dis 2011;13:e252-e255. 
9. Eng OS, Tsang AT, Moore D, et al. Outcomes of microwave ablation for colorectal cancer liver metastases: A single center experience. J Surg Oncol 2015;111:410-413.

10. Wright AS, Sampson LA, Warner TF, Mahvi DM, Lee FT Jr. Radiofrequency versus Microwave Ablation in a Hepatic Porcine Model. Radiology 2005;236:132-136.

11. Veltri A, Gazzera C, Barrera M, et al. Radiofrequency thermal ablation (RFA) of hepatic metastases (METS) from breast cancer $(\mathrm{BC})$ : an adjunctive tool in the multimodal treatment of advanced disease. Radiol Med 2013;119:327333.

12. Öberg K, Knigge U, Kwekkeboom D, Perren A; ESMO Guidelines Working Group. Neuroendocrine gastro-enteropancreatic tumors: ESMO clinical practice guidelines for diagnosis, treatment and follow-up. Ann Oncol 2012;23 Suppl 7:vii124-vii130.

13. Mohan H, Nicholson P, Winter DC, et al. Radiofrequency ablation for neuroendocrine liver metastases: A systematic review. J Vasc Interv Radiol 2015;206:935-942.

14. Guner A, Son T, Cho I, et al. Liver-directed treatments for liver metastasis from gastric adenocarcinoma: comparison between liver resection and radiofrequency ablation. Gastric Cancer 2016;19:951-960.

15. Doussot A, Nardin C, Takaki H, et al. Liver resection and ablation for metastatic melanoma: A single center experience. J Surg Oncol 2015;111:962-968.

16. Ahmed M, Solbiati L, Brace CL, et al. Image-guided Tumor Ablation: Standardization of Terminology and Reporting Criteria-A 10-Year Update. Radiology 2014;25:16911705.

17. Goldberg SN, Grassi CJ, Cardella JF, et al. Image-guided tumor ablation: Standardization of terminology and reporting criteria. J Vasc Interv Radiol 2005;235:728-739.

18. Shady W, Petre EN, Gonen M, et al. Percutaneous radiofrequency ablation of colorectal cancer liver metastases: Factors affecting outcomes-a 10-year experience at a single center. Radiology 2016;278:601-611.

19. Meloni MF, Livraghi T, Filice C, Lazzaroni S, Calliada F, Perretti L. Radiofrequency ablation of liver tumors: the role of microbubble ultrasound contrast agents. Ultrasound Q 2006;22:41-47.

20. Poon RT, Ng KK, Lam CM, et al. Learning curve for radiofrequency ablation of liver tumors: prospective analy- sis of initial 100 patients in a tertiary institution. Ann Surg 2004;239:441-449.

21. Bleicher RJ, Allegra DP, Nora DT, Wood TF, Foshag LJ, Bilchik AJ. Radiofrequency ablation in 447 complex unresectable liver tumors: lessons learned. Ann Surg Oncol 2003;10:52-58.

22. Shiozawa K, Watanabe M, Takayama R, et al. Evaluation of local recurrence after treatment for hepatocellular carcinoma by contrast-enhanced ultrasonography using Sonazoid: comparison with dynamic computed tomography. J Clin Ultrasound 2010;38:182-189.

23. 23. Ierardi AM, Floridi C, Fontana F, et al. Microwave ablation of liver metastases to overcome the limitations of radiofrequency ablation. Radiol Med 2013;118:949961.

24. BO XW, Xu HX, Guo LH, et al. Ablative safety margin depicted by fusion imaging with post-treatment contrastenhanced ultrasound and pre-treatment CECT/CEMRI after radiofrequency ablation for liver cancers. Br J Radiol 2017;90:20170063.

25. Yu J, Liang P, Yu X, Liu F, Chen L, Wang Y. A comparison of microwave ablation and bipolar radiofrequency ablation both with an internally cooled probe: Results in ex vivo and in vivo porcine livers. Eur J Radiol 2011;79:124130 .

26. Bhardwaj N, Strickland AD, Ahmad F, Atanesyan L, West $\mathrm{K}$, Lloyd DM. A comparative histological evaluation of the ablations produced by microwave, cryotherapy and radiofrequency in the liver. Pathology 2009;41:168-172.

27. Calandri M, Yamashita S, Gazzera C, et al. Ablation of colorectal liver metastasis: Interaction of ablation margins and RAS mutation profiling on local tumour progressionfree survival. Eur Radiol 2018;28:2727-2734.

28. Kim KH, Yoon YS, Yu CS, et al. Comparative analysis of radiofrequency ablation and surgical resection for colorectal liver metastases. J Korean Surg Soc 2011;81:25-34.

29. Curley SA, Marra P, Beaty K, et al. Early and late complications after radiofrequency ablation of malignant liver tumors in 608 patients. Ann Surg 2004;239:450-458.

30. Hildebrand P, Leibecke T, Kleemann M, et al. Influence of operator experience in radiofrequency ablation of malignant liver tumours on treatment outcome. Eur J Surg Oncol 2006;32:430-434. 